disease (CHD) at home, although CHD may not be taught in pre-registration paramedic courses. Aim: This study set out to explore how much knowledge student paramedics have surrounding $\mathrm{CHD}$; how prepared they feel for managing children with CHD and the extent to which an online multimedia educational package could enhance the preparedness of paramedic students for managing children with CHD. Method: An action research approach was adopted that firstly identified what paramedic students already knew and how confident they felt in managing these children and their families through a Focus Group $(\mathrm{n}=8)$. An online multimedia learning tool was then developed and made available for the students, who were asked to provide feedback via an online questionnaire after completing the activities. Results: The focus group participants were concerned that they did not know where best to take a child with CHD during an emergency. The group expressed that they had very little knowledge about $\mathrm{CHD}$ and what knowledge they did have had generally been derived from lay sources. Students also expressed concern about interacting with parents in relation to the parent's expectations, the parents' knowledge of their child's condition and transportation issues. All of the focus group participants completed the online multimedia educational package. However only 4 of the participants (50\%) responded to the online evaluation questionnaire despite reminder emails; the results may not therefore be representative of the entire group of participants. Factors impacting upon the completion of the questionnaire will be discussed. Conclusion: There is clearly a need to address paramedic students' knowledge and preparedness for managing children with complex CHD in the pre-hospital setting and communicating effectively with their families. Implications for future development of the educational tool will be discussed.

\section{G31 PROCESS AND OUTCOMES OF THE CAMHS MINISTERIAL TASKFORCE - REPORT AND REFLECTIONS ON THE WAYS IN WHICH CLINICIANS CAN BE INVOLVED IN DEVELOPING EVIDENCE-BASED POLICY}

LJ Baldwin. Nursing/Child and Adolescent Mental Health Services, Derbyshire Healthcare NHSFT, Derby, UK

\subsection{6/archdischild-2015-308599.31}

Aims This session will describe the process by which the CAMHS Ministerial Taskforce was set up and the process of its work, with a recap of the outcomes/recommendations coming from that process. The presentation will also allow some reflections on the political process of policy development and how clinicians can be involved in fostering evidence-based policy in healthcare.

Methods The political background to the process and the ways in which the taskforce worked will be described, along with the outcomes and likely impact on practice.

Results The taskforce will have reported by the time of the conference and the recommendations for government will have been published. These will be recapped, along with some ideas as to how these will influence commissioning for future specialist CAMHS, impact on primary care and paediatrics, and how the education of future health workforce as well as other professional groups such as social work and school teachers is likely to be influenced. Themes of big data, integration of services, early intervention and CAMHS in-patient provision will be covered in some detail, alongside ideas for future work arising from these recommendations.
In wider terms the ways in which government responds to pressure and concerns of clinicians, charities and patients/service users will be examined in terms of the political process and set against some ideas of how clinicians and others can influence the creation of evidence-based healthcare policy. This will be matched against other processes (NICE guidelines for example), with some reflections on how nurses, doctors and other clinicians can be more actively involved in developing policy. In particular whether some groups are better than others at having a voice in the process will be examined, and whether there are ways in which this can be improved for the future, leading to more evidence-based policy and less anecdotally-based, or politically-based policies within healthcare.

Conclusions This session will argue for nurses in particular to have a better voice in the development of evidence-based policies for healthcare. It will look at how all healthcare professionals currently influence policy development and look for ways to improve this process.

\section{G32 AN EVALUATION OF A COMPLEX SIMULATED CLINICAL SHIFT SCENARIO WITH FINAL YEAR UNDERGRADUATE CHILDREN'S NURSING STUDENTS}

D Clarke, J Davies, M Nathan. School of Healthcare Sciences, Cardiff University, Cardiff, UK

\subsection{6/archdischild-2015-308599.32}

Background The last decade has witnessed the emergence of simulation as a useful, but some times challenging teaching methodology. This is supported by a number of sources of evidence, which focus strongly upon student evaluation of the learning experience. The challenges are equally worth consideration. These include issues such as the commissioning of space and staff expertise in resourcing and creating a situation which is as close to real practice as possible.

Aims To explore the use of a complex simulated scenario with third year children's nursing students.

To share the process of educational evaluation that was undertaken.

To share the results of this educational intervention.

Methods Children's nursing students were exposed to a complex simulated clinical shift. The activities included: managing the clinical area; the transfer of a sick child; managing a medication error; subsequent discussion with the parents; and prioritising and organising care. The use of role-play was utilised throughout, to create a realistic experience and the simulation activity took place in two settings to enable the transfer of a child from one clinical area (the assessment unit) area to another (the ward).

The simulation experience was undertaken at the end of the third year undergraduate program by three cohorts of students $(n=41)$. Following the experience, data were collected through a post-simulation de-briefing and a Likert style questionnaire, enabling the collection of qualitative and quantitative evaluation data concerned with the student's experience of the simulation. Results and conclusion Overwhelmingly, the results showed that this was a positive experience for the students, demonstrating a number of perceived improvements in the application of their clinical skills. These included: development of specific management skills; enhanced confidence; development of self-awareness; and the transferability of skills to practice. Students also demonstrated strong agreement in terms of the realism of the experience. 